
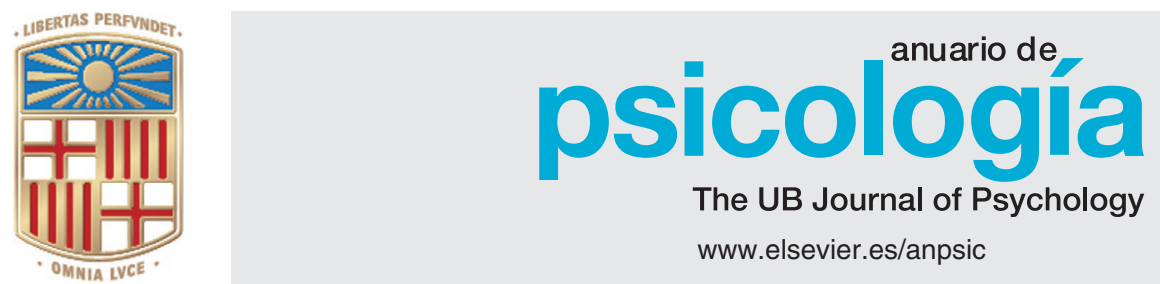

ARTÍCULO DE INVESTIGACIÓN

\title{
Desarrollo y desempeño en equipos de proyecto: validez incremental de la escala de desarrollo grupal
}

\author{
José Navarro ${ }^{\mathrm{a}, \mathrm{b}, *}$, Rocío Meneses ${ }^{\mathrm{a}}$, Miquel Nadal ${ }^{\mathrm{a}}$ y Edwald Landsberger ${ }^{\mathrm{a}}$
}

a Departamento de Psicología Social, Facultad de Psicología, Universitat de Barcelona, Barcelona, España

${ }^{\mathrm{b}}$ Grup de Recerca en Psicologia Social, Ambiental i Organitzacional (2014SRG992), Universitat de Barcelona, Barcelona, España

Recibido el 21 de enero de 2016; aceptado el 02 de marzo de 2016

\section{PALABRAS CLAVE}

Equipos de trabajo; Desarrollo del grupo; Desempeño del equipo; Validez incremental

\section{KEYWORDS}

Teams;

Group development;

Team performance;

Incremental validity

\begin{abstract}
Resumen Se presenta un estudio acerca de cómo el desarrollo alcanzado por un grupo es un determinante clave en el desempeño de equipos de proyecto. Se aplicó la Escala de Desarrollo del Grupo (EDG) y el Group Development Questionnaire (GDQ) a 30 equipos de proyecto y utilizando diferentes criterios subjetivos para evaluar el desempeño de los mismos (i. e. valoración de eficacia, satisfacción de necesidades, funcionamiento interno y mantenimiento). Mediante la realización de un estudio transversal, y controlando por el tamaño del grupo, el desarrollo grupal se mostró capaz de predecir algunos de estos criterios de desempeño, como la valoración de eficacia ( $23 \%$ de varianza explicada) y la satisfacción de necesidades (53\% de varianza explicada). Igualmente, y respecto a una medida global de desempeño del equipo, el desarrollo grupal explicó el $40 \%$ de su varianza. Además, la EDG mostró validez incremental respecto al GDQ $(F=5,84, p<0,01)$ para predecir el desempeño. Se discute también cómo contribuir al desarrollo grupal como vía para lograr equipos más efectivos.

(c) 2016 Universitat de Barcelona. Publicado por Elsevier España, S.L.U. Todos los derechos reservados.
\end{abstract}

Development and performance in team projects: Incremental validity of the group development scale

\begin{abstract}
We carry out a study about how the development achieved by a group is a key determinant of the performance in project teams. The group development scale (EDG) and the group development questionnaire (GDQ) were applied to 30 project teams using different subjective criteria to evaluate their performance (i.e. self-reported effectiveness, satisfaction of needs' members, internal operations and maintenance). By carrying out a cross sectional study, and controlling for group size, group development was able to predict some of these criteria of performance such as self-reported effectiveness ( $23 \%$ of explained variance) and the satisfaction of needs ( $53 \%$ of variance explained). Similarly, with regard to an overall measure of team performance, group development accounted for $40 \%$ of its variance. In addition, the EDG
\end{abstract}

*Autor para correspondencia.

Correo electrónico: j.navarro@ub.edu (J. Navarro). 
showed incremental validity relative to $\operatorname{GDQ}(F=5,84, p<.01)$ to predict team performance. Finally, we also discuss how to contribute to group development as a way to achieve more effective teams.

(c) 2016 Universitat de Barcelona. Published by Elsevier España, S.L.U. All rights reserved.

El constructo ‘desarrollo del grupo' ha sido ampliamente investigado y posee un amplio respaldo empírico (Wheelan, 1999, 2005). Ha sido propuesto en los clásicos modelos de fases (e.g., Tuckman y Jensen, 1977; Wheelan, 1994) y utilizado también por una línea de investigación distinta centrada en mostrar qué características permiten diferenciar un auténtico grupo de meros agregados sociales (Meneses, Ortega, Navarro y De Quijano, 2008). Según este último enfoque, que llamaremos sencillamente 'desarrollo grupal' (DG), este desarrollo se caracteriza por cuatro elementos básicos: interrelación entre sus miembros, identificación con el grupo, coordinación de comportamientos, recursos, esfuerzos y herramientas, y orientación del comportamiento hacia la consecución de metas compartidas (e.g., Arrow, McGrath y Berdhal, 2000; Lickel et al., 2000; Meneses et al., 2008). A mayor presencia de estas propiedades mayor desarrollo muestra el grupo y mayor puede ser su efectividad (Meneses y Navarro, 2015; Navarro, Meneses, Miralles, Moreno y Loureiro, 2015). Para ambos enfoques el desarrollo está relacionado con otros procesos y con el desempeño.

La relación entre procesos grupales, entre ellos el desarrollo, y el desempeño ha sido bastante estudiada y, aunque no todos los resultados coinciden, múltiples investigaciones la avalan (e.g., Gully, Incalcaterra, Joshi y Beaubien, 2002; LePine, Piccolo, Jackson, Mathieu y Saul, 2008; Srivastava, Bartol y Locke, 2006). Específicamente, en relación con el desarrollo del grupo diferentes autores han encontrado que este se relaciona positivamente tanto con el desempeño como con la efectividad del equipo (e.g. Navarro et al., 2015; Wheelan, 2009). Por ejemplo, en la investigación desarrollada por Navarro et al. (2015) el desarrollo del grupo predijo el desempeño del grupo, así como los niveles de absentismo dentro del equipo y el orden de materiales e higiene en equipos de producción de la industria automovilística. Además, para el caso de los equipos de proyecto en los que aquí nos centraremos el desarrollo alcanzado se ha mostrado particularmente importante para determinar el desempeño de los equipos. En efecto, tras los estudios desarrollados por Gersick $(1988,1989)$ sabemos que, en este tipo de equipos, que se organizan en torno a la realización de unas tareas orientadas al logro de un proyecto y en los que hay una fecha de finalización, el desarrollo no sigue un proceso lineal sino que muestra un salto cualitativo importante justo al atravesar la vida media del grupo a partir del cual el equipo se centra más en la tarea y consigue entonces desempeñarse con más eficacia. Por otro lado, en un meta-análisis realizado considerando 93 estudios, Stewart (2006) aporta evidencia clara sobre cómo algunos procesos grupales, por ejemplo la coordinación, es determinante en el desempeño de estos equipos.

Nuestro objetivo en la presente investigación será estudiar la capacidad que tiene el desarrollo grupal (DG) para explicar resultados en equipos de proyecto. A continuación, describiremos los modelos teóricos que utilizaremos, el mo- delo de fases de Wheelan y el modelo de DG de Meneses et al., para pasar después a describir los objetivos específicos perseguidos en esta investigación.

\section{Modelo de desarrollo de fases de Wheelan}

El modelo de desarrollo de fases de Wheelan (1994, 2005) ofrece una visión global y lineal del desarrollo del equipo, describe patrones de cambio normativos que implican una progresión a través de etapas consecutivas definidas en función de la proporción de tiempo que un grupo invierte en resolver los aspectos característicos de cada etapa e independientemente de otras características del grupo (e.g., tamaño, composición, etc.). Cada etapa se define según los comportamientos predominantes durante ese lapso específico, no estableciéndose límites precisos entre una fase y la siguiente (Chang, Bordia y Duck, 2003; Chang, Duck y Bordia, 2006).

Este modelo señala también el carácter dinámico y continuo del desarrollo, ayudando a comprender los cambios en la estructura y procesos del grupo. De acuerdo con su autora, los grupos pasarán por cinco etapas, cada una caracterizada por un determinado patrón de comportamiento y procesos grupales. En general, los grupos pueden desarrollarse, retroceder o detenerse; y pueden comportarse en forma saludable y madura o patológica y destructiva. Siguiendo las propuestas de Wheelan (e.g., Wheelan, 2005, 2009; Wheelan y Hochberger, 1996) las características más relevantes de cada fase serían:

Etapa I: Dependencia e inclusión. Los grupos dirigen su energía principalmente hacia asuntos relacionados con la dependencia, seguridad e inclusión. A menudo, sus miembros sienten tensión, ansiedad e inseguridad, en relación con sí mismos, al grupo y a su estructura, por lo nuevo e indefinición de la situación. Se sienten dependientes del líder y tienden a estar de acuerdo con las propuestas del grupo. La comunicación entre los miembros y sus actividades enfatizan encontrar sentido a la nueva situación que supone el grupo. El nivel de productividad suele ser bajo, el grupo aún no está listo para trabajar y sus miembros necesitan adquirir seguridad dentro éste para pasar a la etapa siguiente.

Etapa II: Contra-dependencia y lucha. Los miembros se centran en cuestiones relativas al conflicto y la contra-dependencia. Son frecuentes los desacuerdos entre ellos y en relación con el líder, a las metas grupales, normas y procedimientos. Tratan de clarificar sus roles y el grupo busca reafirmar su independencia frente al líder. Pueden surgir subgrupos y coaliciones. Si se manejan adecuadamente los conflictos aumenta la confianza y el compromiso con el grupo, la cohesión y la cooperación. La comunicación se hace más abierta y más orientada a la tarea; comienzan a establecerse valores y normas compartidas. 
Etapa III: Confianza y estructura. El grupo se focaliza en el establecimiento de la confianza y la estructura del grupo. Se negocian roles, aspectos relativos a la organización y procedimientos a seguir. Se consolidan las relaciones entre los miembros y con el líder. Se logra mayor consenso hacia las normas y las metas del grupo. Aumenta la productividad, confianza, cohesión y satisfacción de los miembros y se hace posible el feedback.

Etapa IV: Trabajo. El grupo dirige su energía fundamentalmente hacia el logro de tareas y metas, es productivo y efectivo. Si la producción es baja, probablemente sea debido a que el grupo se haya atascado en una de las etapas previas del desarrollo. La comunicación es más abierta y efectiva. El grupo hace un uso más provechoso de los recursos disponibles y del tiempo; trabaja en su tarea fundamental sobre un $60 \%$ del tiempo y el resto lo dedica a su mantenimiento. En las fases previas los grupos también trabajan en la consecución de los objetivos asignados, pero con un porcentaje de tiempo dedicado menor.

Etapa V: Finalización. Los grupos de trabajo al concluir la tarea asignada llegan a su final, aun cuando los miembros puedan proseguir luego con otros proyectos y conformen nuevos equipos. Aquí los grupos funcionales tienden a evaluar su trabajo en conjunto, dar feedback y expresar sentimientos hacia los otros miembros y hacia el grupo (e.g., acciones relacionadas con una despedida simbólica). También puede ocurrir que el grupo regrese a las primeras etapas mostrando tensión, conflicto o mala comunicación.

Wheelan y colaboradores (Wheelan y Hochberger, 1996), además de una herramienta observacional (el GDOS o Group Development Observation System), han desarrollado y validado una medida de auto-informe para medir el desarrollo del grupo en cada una de las fases (el GDQ o Group Development Questionnaire). Consta de 60 ítems en un formato de respuesta Likert con rango de 1-5, distribuidos equitativamente en cuatro escalas correspondientes a las cuatro primeras etapas del modelo y miden el nivel de presencia-ausencia de los comportamientos característicos en cada fase. La quinta etapa (Finalización) no está incluida porque su objetivo es evaluar grupos en funcionamiento. El instrumento está respaldado por múltiples estudios de validez y fiabilidad (Buzzaglo y Wheelan, 1999; Wheelan, 2009; Wheelan, Davidson y Tilin, 2004; Wheelan y Hochberger, 1996). Este cuestionario no ha sido publicado en su totalidad, pero sí se han publicado repetidamente tres ítems por escala de los que también existe versión castellana utilizada por Buzaglo y Wheelan (1999).

\section{Modelo de desarrollo grupal de Meneses, Navarro et al.}

El constructo de desarrollo grupal también cuenta con una sólida base teórica y empírica. Un análisis de revisión teórica realizado por Meneses et al. (2008) puso de relieve varios aspectos importantes al respecto. Primero, que el concepto de desarrollo grupal ha contado con una larga tradición en psicología social que se remonta a la década de los 50 del siglo pasado y que ha continuado hasta nuestros días bajo el paraguas de tres corrientes teóricas que han hablado de groupness, entitativity (entitatividad) y grupalidad. Segundo, que estas tres corrientes con orígenes distintos, psicolo- gía de los grupos, psicología de la percepción y psicología soviética respectivamente, comparten la idea de continuo entre el no grupo y el grupo. Comparten, por tanto, la consideración del grupo como un fenómeno borroso en el que se puede hablar de grados de grupalidad, tal y como fuera propuesto hace décadas por McGrath (1984). Y tercero, y ha sido el aporte peculiarmente significativo del trabajo de Meneses et al. (2008), que pueden identificarse una serie de criterios compartidos por estas diferentes corrientes teóricas y que nos permitirían la caracterización básica y fundamental de todo grupo humano, también de los grupos y equipos de trabajo. A saber, en todo grupo encontramos las siguientes cuatro características: 1) interrelación entre los miembros (i. e., interacción social frecuente entre los miembros); 2) identificación con el grupo (i. e., sentido de pertenencia al grupo desarrollado por los miembros); 3) coordinación (i. e., desarrollo de mecanismos de coordinación, de sincronización, de recursos, herramientas y esfuerzos individuales puestos al servicio de los objetivos del grupo); y 4) orientación hacia la consecución de las metas del grupo (i. e., dirección de los comportamientos individuales a favor del logro de las metas y objetivos del grupo). En resumen, el desarrollo grupal sería un ejemplo de estado emergente (Marks, Mathieu y Zaccaro, 2001; Rico, Alcover y Tabernero, 2010) y, por tanto, mediador entre las entradas (e.g., las tareas a realizar o la composición del equipo) y los resultados conseguidos (e.g. la efectividad alcanzada).

Posteriormente, Navarro et al. (2015) han aportado resultados sobre la creación de una herramienta unidimensional (la escala de desarrollo grupal o EDG) compuesta por ocho ítems tipo Likert con rango 1-5 que permite la evaluación del desarrollo del grupo desde este enfoque teórico. Los resultados mostrados en diferentes estudios con cientos de grupos reales de trabajo han mostrado unos buenos resultados de validez (de contenido, factorial, de criterio y convergente) así como unos índices de fiabilidad satisfactorios (valores alfa entre 0,70 y 0,85 ) y una capacidad predictiva significativa respecto a indicadores concretos de efectividad como el absentismo y el orden e higiene en el lugar de trabajo, y respecto al propio desempeño del equipo (ver también Meneses y Navarro, 2015).

\section{Objetivos del presente estudio}

Con el objetivo de dar continuidad a la literatura anterior (e.g., Buzaglo y Wheelan, 1999; Gully et al., 2002; Navarro et al., 2015; Wheelan, 2009), interesada en conocer las influencias del desarrollo grupal como estado emergente que determina el desempeño alcanzado por el grupo, estudiaremos aquí la relación entre estas variables en equipos de proyecto. Esperamos encontrar, tal y como ya ha sido descubierto por Gersick $(1988,1989)$ utilizando un enfoque de desarrollo grupal diferente al que emplearemos aquí, que el desarrollo grupal incide de manera significativa en el desempeño eficaz del grupo de trabajo. Como apuntábamos arriba centrase en equipos de proyecto es interesante por sus características diferenciales respecto a otros equipos (e.g., temporalidad limitada, alta interdependencia, etc.) y porque en estos equipos su desarrollo parece ser un proceso que determina de manera especial lo eficaces que llegan a ser. 
Los objetivos específicos de la presente investigación serán, por tanto, los siguientes:

1. Estudiar las relaciones entre el desarrollo y el desempeño en equipos de proyecto.

2. Comparar dos modelos de desarrollo grupal, los modelos de Wheelan (e.g., Wheelan, 2009) y de Meneses et al. (e.g. Meneses et al., 2008; Navarro et al., 2015), en cuanto a su capacidad de predicción del desempeño de equipos de proyecto.

\section{Método}

\section{Participantes y procedimiento}

Partimos de la siguiente definición de grupo de trabajo: grupos formales de una organización, cuyos miembros se hallan bajo la supervisión de un mismo jefe, tienen un tamaño limitado (3-15 miembros) y comparten metas asignadas por la organización. La muestra estuvo compuesta por 30 grupos de proyecto pertenecientes a diferentes organizaciones del sector público y privado. Tuvieron 3,6 miembros de promedio (rango 3-9) y un tiempo promedio de pertenencia al grupo de 4 meses. En el caso de pertenencia múltiple los participantes solo se identificaban como miembros del grupo al que dedicaran más tiempo o en el que consideraban más importante el rol desempeñado. Se contactó con los responsables de recursos humanos de las organizaciones para conseguir su implicación en la investigación y para que nos permitieran el acceso a los grupos de trabajo. Luego se explicó a los grupos el estudio a realizar y su importancia; garantizamos la confidencialidad y anonimato de las respuestas a los participantes. Una vez procesados los datos les proporcionamos a cada organización y a los grupos participantes un informe con sus resultados.

\section{Instrumentos}

Aplicamos tres instrumentos que tenían un total de 32 ítems, distribuidos en tres escalas. Dos de ellas medían el desarrollo grupal y la otra el desempeño que siempre se presentaron en el mismo orden y agrupados por variable medida (desarrollo y desempeño). En todos los cuestionarios utilizamos una escala tipo Likert (rango 1 «Muy en desacuerdo» a 5 «Muy de acuerdo»).

Las dos herramientas para medir el desarrollo grupal (variable predictora), fueron la escala de desarrollo grupal (EDG, Navarro et al., 2015) y una versión reducida del GDQ original de Wheelan (Wheelan y Hochberger, 1996). Ejemplos de los ocho ítems de la EDG son: «Tenemos una forma habitual de funcionar como grupo», «Compartimos herramientas, recursos e información». Esta escala mostró un adecuado valor de fiabilidad $(\alpha=0,84)$. Por su lado, la versión del GDQ que utilizaremos aquí (Buzaglo y Wheelan, 1999) cuenta con 12 ítems, entre ellos: Los miembros tienen una tendencia a hacer lo que es sugerido por el líder» (Fase I), «Los miembros parecen tener opiniones muy diferentes en cuanto a cómo se tienen que hacer las cosas en este grupo» (Fase II), «El grupo está pasando tiempo pla- neando como va a implementar su tarea» (Fase III) y «El grupo recibe, da y utiliza retroalimentación sobre su efectividad y productividad» (Fase IV). La escala mostró igualmente un adecuado valor de fiabilidad $(\alpha=0,70)$. En sus estudios, Wheelan (e.g., Wheelan, 2009) ha solido utilizar la puntuación en la Fase IV como predictor de la efectividad, procedimiento que también seguimos nosotros. Además, en nuestro caso, creamos también una medida única del GDQ ${ }^{1}$ fundamentada en las puntuaciones de las cuatro fases.

Para medir el desempeño utilizamos la escala creada por Navarro, Quijano, Berger y Meneses (2011) basada en los modelos de Hackman (1987) y McGrath y Argote (2001) que considera cuatro criterios: valoración de eficacia, satisfacción de necesidades individuales, funcionamiento interno y mantenimiento del grupo. La escala tiene 12 ítems en formato tipo Likert (rango 1-5) y también permite obtener un índice general de efectividad (EFG). Algunos ejemplos de ítems son: «Somos eficaces realizando nuestras tareas» (valoración de eficacia), «Con frecuencia las necesidades de los miembros pasan desapercibidas» (satisfacción de necesidades, inverso), «Funcionamos bien como equipo de trabajo» (funcionamiento interno) y «Las incorporaciones de nuevos miembros se valora positivamente» (mantenimiento del grupo). La medida general EFG también mostró un valor adecuado de fiabilidad $(\alpha=0,87)$.

\section{Diseño de investigación y análisis de datos}

Utilizamos un diseño transversal y correlacional. Dado que estamos considerando constructos grupales y que las medidas se obtienen a partir de las percepciones individuales de los miembros del grupo, calculamos el índice de acuerdo intra-grupo ADM basado en la mediana dado el número pequeño de miembros por grupo (median average deviation; ver Cohen, Doveh y Nahum-Shani, 2009). Realizamos análisis descriptivos, correlaciones entre las variables, estudios de fiabilidad de las escalas y para evaluar la capacidad de influencia de las variables predictoras (EDG, Fase IV y GDQ) en el criterio (EFG) empleamos la regresión jerárquica (Hunsley y Meyer, 2003). Incluimos como variable control en estos últimos análisis el tamaño del grupo ya que es conocida su influencia en las relaciones objeto de estudio en este trabajo (e.g., Wheelan, 2009).

\section{Resultados}

Presentamos, primero, los principales resultados descriptivos. En la tabla 1 pueden verse, además de otros resultados, los valores promedios y desviaciones. Los valores promedios oscilan entre 2,80 del funcionamiento interno y 3,63 del tamaño del grupo. La EDG obtiene un valor promedio de 3,62, muy parecido, aunque algo superior, al obtenido por la GDQ y por la GDQ - Fase IV. Todas las medidas, excepto la sub-

1 La formulación para la construcción de esta medida GDQ trato de ponderar la importancia de las diferentes fases para el desarrollo del grupo de la siguiente manera: GDQ $=(($ Fase I * 1$)+($ Fase II * 2$)+$ $($ Fase III *3) $+($ Fase IV * 4) $) / 10$ 
escala de EFG sobre funcionamiento interno, muestran valores ligeramente superiores al promedio teórico de las escalas (igual a 3) indicando cierta asimetría en estas distribuciones de datos.

En la tabla 1 también recogemos los resultados de los índices de acuerdo (ADM) que, en general, mostraron valores inferiores a los proporcionados por las simulaciones siguiendo el procedimiento descrito por Cohen et al. (2009); esto significa que todos los valores encontrados resultaron significativos $(p<0,01)$ indicando acuerdo intra-grupal en cuanto a las variables medidas, por lo que la agregación de medidas era procedente y podíamos hablar de una puntuación grupal. También recogemos los índices de correlación intra-clase (ICC1 e ICC2), de interés en este tipo de estudio por cuanto nos indican la varianza atribuible a la pertenencia a un grupo (ICC[1]) y la fiabilidad para diferenciar a los grupos entre sí (ICC[2]). Los resultados aquí indican poca varianza explicada por el hecho de pertenecer a un grupo (valores entre 0,03 y 0,22 ) así como unos valores de fiabilidad medios para diferenciar entre grupos (valores en torno a 0,4 ).

Los resultados del análisis de fiabilidad de las herramientas se situaron en torno al 0,70 , valor adecuado según las convenciones establecidas (ver diagonal de la tabla 1). Solo se encontraron valores inferiores para las escalas de Fase IV y la sub-escala de EFG sobre funcionamiento interno. Estos resultados indican alta consistencia interna en la mayoría de las escalas utilizadas y fiabilidad moderada para las dos últimas mencionadas.

Por último, y para cerrar los resultados presentados en la tabla 1, la mayoría de las medidas mostraron correlaciones significativas entre ellas y algunas fueron especialmente altas. La correlación entre EDG y GDQ $(0,74)$ y EDG y GDQ Fase IV $(0,79)$ indicaría que están midiendo el mismo constructo (desarrollo grupal). Un AFC (método ML) mostró que un modelo de dos factores que diferenciara entre los ítems de EDG y los de GDQ no obtenía resultados significati- vamente mejores que el modelo nulo que representaría la no diferenciación de las dos medidas $(\mathrm{CFI}=0,62 ; \mathrm{TLI}=$ $0,57)$. También es de destacar las relaciones entre las cuatro sub-escalas de desempeño en las que la medida de funcionamiento interno muestra un patrón de relaciones distinto al resto. Por último, el tamaño del grupo no mostró valores significativos con la mayoría de las variables estudiadas.

En la figura 1 se representa gráficamente la relación entre la puntuación obtenida por la EDG y las fases propuestas por Wheelan (siguiendo el procedimiento de cálculo propuesto por la propia Wheelan según la cual el grupo se halla en la fase de desarrollo que muestra un mayor valor). Los resultados muestran una clara relación entre ambas medidas según lo esperado, útil para asignar valor numérico al desarrollo grupal en cada una de las fases descritas por Wheelan. Además, la medida global GDQ generada mostró una correlación muy clara $(r=0,90, p<0,01)$ con la fase en la que se encuentra el grupo (siguiendo como hemos dicho el cálculo propuesto por Wheelan).

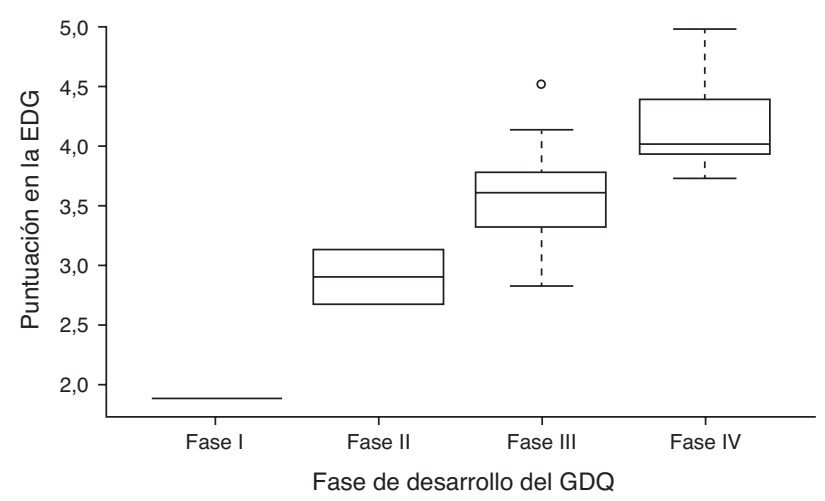

Figura 1 Relación entre la medida del EDG y las Fases de Desarrollo propuestas por Wheelan.

Tabla 1 Estadísticos descriptivos, índices de acuerdo y de correlación intra-clase, y correlaciones entre las diferentes medidas utilizadas

\begin{tabular}{|c|c|c|c|c|c|c|c|c|c|c|c|c|c|c|}
\hline & M & SD & ADM & $\operatorname{ICC}(1)$ & $\operatorname{ICC}(2)$ & 1 & 2 & 3 & 4 & 5 & 6 & 7 & 8 & 9 \\
\hline 1. EDG & 3,62 & 0,62 & 0,58 & 0,17 & 0,43 & $(0,84)$ & & & & & & & & \\
\hline 5. GDQ - Fase IV & 3,58 & 0,60 & 0,59 & 0,22 & 0,51 & $0,79^{* *}$ & $(0,60)$ & & & & & & & \\
\hline 3. GDQ & 3,37 & 0,33 & 0,60 & 0,03 & 0,12 & $0,74^{* *}$ & $0,83^{* *}$ & $(0,70)$ & & & & & & \\
\hline $\begin{array}{l}\text { 4. Valoración } \\
\text { eficacia }\end{array}$ & 3,22 & 0,55 & 0,47 & 0,14 & 0,37 & $0,48^{* *}$ & $0,44^{*}$ & 0,26 & $(0,75)$ & & & & & \\
\hline $\begin{array}{l}\text { 5. Satisfacción } \\
\text { de necesidades }\end{array}$ & 3,16 & 0,49 & 0,61 & 0,08 & 0,26 & $0,76^{* *}$ & $0,62^{* *}$ & $0,62^{* *}$ & $0,27 \mathrm{~ns}$ & $(0,75)$ & & & & \\
\hline $\begin{array}{l}\text { 6. Funcionamiento } \\
\text { interno }\end{array}$ & 2,80 & 0,35 & 0,55 & 0,22 & 0,51 & $-0,20 \mathrm{~ns}$ & $-0,23 n s$ & $-0,12 n s$ & $-0,18 n s$ & $-0,27 n s$ & $(0,57)$ & & & \\
\hline 7. Mantenimiento & 3,50 & 0,51 & 0,63 & 0,15 & 0,40 & $0,28 \mathrm{~ns}$ & $0,33 \mathrm{~ns}$ & $0,19 \mathrm{~ns}$ & $0,61^{* *}$ & $0,08 \mathrm{~ns}$ & $-0,43^{*}$ & $(0,64)$ & & \\
\hline 8. EFG & 3,17 & 0,27 & 0,57 & 0,15 & 0,40 & $0,67^{* *}$ & $0,60^{* *}$ & $0,47^{* *}$ & $0,87^{* *}$ & $0,55^{* *}$ & $-0,10 \mathrm{~ns}$ & $0,69^{* *}$ & $(0,87)$ & \\
\hline $\begin{array}{l}\text { 9. Tamaño } \\
\text { del grupo }\end{array}$ & 3,63 & 2,19 & ND & ND & ND & $-0,12 \mathrm{~ns}$ & $-0,27 \mathrm{~ns}$ & $-0,14 \mathrm{~ns}$ & $-0,30 \mathrm{~ns}$ & $-0,21 \mathrm{~ns}$ & $0,40^{*}$ & $-0,23 n s$ & $-0,23 \mathrm{~ns}$ & (ND) \\
\hline
\end{tabular}

ND: no disponible; ns: no significativo.

$\mathrm{N}=30$ grupos.

Entre paréntesis, en la diagonal, recogemos los valores alfa de Cronbach.

${ }^{*} p<0,05,{ }^{* *} p<0,01$. 
En la tabla 2 presentamos la comparación entre los diferentes modelos de regresión que utilizamos en el estudio de la relación entre el desarrollo y el desempeño considerando además como variable de control el tamaño del grupo (Modelo 0). En primer lugar, en el Modelo 1 recogemos la relación con el desempeño, y sus diferentes indicadores, del GDQ - Fase IV y GDQ. Según los resultados, el desarrollo grupal explicaría el 29,13\% del desempeño del equipo, el $17,02 \%$ de la valoración de eficacia y el $36,33 \%$ de la satisfacción de necesidades. Por otra parte, la relación con las otras dos medidas de eficacia, funcionamiento interno y mantenimiento del grupo, no sería significativa. Ahora bien, los dos predictores incluidos solo resultan significativos en una de las ocasiones (el GDQ - Fase IV y respecto a la medida global de desempeño; $\beta=2,14, p<0,05$ ).

En segundo lugar, y en el Modelo 2, añadimos la variable EDG. El análisis de colinealidad al incluir una nueva variable independiente produjo estimaciones estables, lo que permiten considerar la influencia de cada una de las variables predictoras de manera independiente. Las puntuaciones en las escalas EDG, GDQ y GDQ - Fase IV muestran un adecuado nivel de tolerancia $y$, aunque cada una se relaciona con el desempeño de los grupos, el porcentaje de predicción compartida es bajo por lo que no aportan información redundante. Al comparar los resultados de este Modelo 2 con respecto al Modelo 1 previo vemos que la capacidad de predicción del desarrollo grupal respecto al desempeño del gru- po ha mejorado. En efecto, ahora el desarrollo grupal explicaría el 40,2\% del desempeño el equipo, el 23,7\% de la valoración de eficacia y el $53,7 \%$ de la satisfacción de necesidades. Además, la EDG muestra unos valores beta significativos en todos estos casos. Nuevamente, la relación respecto a las otras dos medidas de desempeño, funcionamiento interno y mantenimiento, no resulta significativa.

El incremento de varianza explicada del Modelo 2 respecto al Modelo 1 nos lleva a preguntarnos si estas diferencias resultarían significativas o no. Un análisis de varianza comparando ambos modelos reveló que efectivamente lo era $\left(F_{\mathrm{EFG}}=5,84, p<0,01 ; F_{\text {eficacia }}=3,27, p<0,05 ; \mathrm{y} F_{\text {satisfacción }}=\right.$ $10,78, p<0,01)$. En cuanto que tanto la GDQ como la EDG miden el mismo proceso, el desarrollo grupal, estos resultados nos estarían indicando que la EDG mostraría una validez incremental respecto al GDQ. En la figura 2 representamos estas diferencias.

En resumen, el desarrollo grupal, especialmente tal y como es evaluado por la EDG, sería un predictor del desempeño eficaz del grupo de trabajo y de dos de sus indicadores fundamentales, valoración de eficacia y satisfacción de necesidades de los miembros, en los grupos de proyecto. Por el contrario, el desarrollo del grupo no ha mostrado relación con las medidas de funcionamiento interno y de mantenimiento de dichos grupos. Tampoco el tamaño del grupo se mostró como un factor relevante en el desempeño de los equipos de proyecto.

Tabla 2 Comparación de diferentes modelos de regresión respecto al desempeño del equipo

\begin{tabular}{|c|c|c|c|c|c|c|c|c|c|c|}
\hline & \multicolumn{2}{|l|}{ EFG } & \multicolumn{2}{|c|}{ Valoración eficacia } & \multicolumn{2}{|c|}{$\begin{array}{l}\text { Satisfacción } \\
\text { de necesidades }\end{array}$} & \multicolumn{2}{|c|}{$\begin{array}{l}\text { Funcionamiento } \\
\text { interno }\end{array}$} & \multicolumn{2}{|c|}{ Mantenimiento } \\
\hline & $\beta$ & $\mathrm{t}$ & $\beta$ & $\mathrm{t}$ & $\beta$ & $\mathrm{t}$ & $\beta$ & $\mathrm{t}$ & $\beta$ & $\mathrm{t}$ \\
\hline \multicolumn{11}{|l|}{ Modelo 0} \\
\hline Intercepto & 3,2754 & $34,49^{* *}$ & 3,4953 & $18,28^{* *}$ & 3,3313 & $18,92^{* *}$ & 2,5748 & $22,31^{* *}$ & 3,6928 & $20,31^{* *}$ \\
\hline \multirow[t]{3}{*}{ Tamaño grupo } & $-0,0286$ & $-1,274$ & $-0,0766$ & $-1,692$ & $-0,0469$ & $-1,12$ & 0,0629 & $2,30^{*}$ & $-0,0539$ & $-1,25$ \\
\hline & \multicolumn{2}{|c|}{$\mathrm{R}^{2}$ adj. $=0,0210$} & \multicolumn{2}{|c|}{$\mathrm{R}^{2}$ adj. $=0,0604$} & \multicolumn{2}{|c|}{$\mathrm{R}^{2}$ adj. $=0,0091$} & \multicolumn{2}{|c|}{$\mathrm{R}^{2}$ adj. $=0,1293$} & \multicolumn{2}{|c|}{$\mathrm{R}^{2}$ adj. $=0,0192$} \\
\hline & \multicolumn{2}{|c|}{$F(1,28)=1,623$} & \multicolumn{2}{|c|}{$F(1,28)=2,864$} & \multicolumn{2}{|c|}{$F(1,28)=1,268$} & \multicolumn{2}{|c|}{$F(1,28)=5,306^{*}$} & \multicolumn{2}{|c|}{$F(1,28)=1,569$} \\
\hline \multicolumn{11}{|l|}{ Modelo 1} \\
\hline Intercepto & 2,3561 & $4,97^{* *}$ & 2,9330 & $2,78^{* *}$ & 0,5143 & 0,62 & 2,6183 & $3,77^{* *}$ & 3,3588 & $3,20^{* *}$ \\
\hline Tamaño grupo & $-0,0088$ & $-0,440$ & $-0,0438$ & $-0,98$ & $-0,0167$ & $-0,47$ & 0,0554 & $1,88^{*}$ & $-0,0302$ & $-0,68$ \\
\hline GDQ - Fase IV & 0,2778 & $2,14^{*}$ & 0,5808 & 2,01 & 0,2458 & 1,08 & $-0,1438$ & $-0,75$ & 0,4287 & 1,49 \\
\hline \multirow[t]{3}{*}{ GDQ } & $-0,0444$ & $-0,194$ & $-0,4862$ & $-0,95$ & 0,5426 & 1,35 & 0,1481 & 0,44 & $-0,3825$ & $-0,75$ \\
\hline & \multicolumn{2}{|c|}{$\mathrm{R}^{2}$ adj. $=0,2913$} & \multicolumn{2}{|c|}{$\mathrm{R}^{2}$ adj. $=0,1702$} & \multicolumn{2}{|c|}{$\mathrm{R}^{2}$ adj. $=0,3633$} & \multicolumn{2}{|c|}{$\mathrm{R}^{2}$ adj. $=0,0864$} & \multicolumn{2}{|c|}{$\mathrm{R}^{2}$ adj. $=0,0527$} \\
\hline & \multicolumn{2}{|c|}{$F(3,26)=4,973^{* *}$} & \multicolumn{2}{|c|}{$F(3,26)=2,982^{*}$} & \multicolumn{2}{|c|}{$F(3,26)=6,516^{* *}$} & \multicolumn{2}{|c|}{$F(3,26)=1,914$} & \multicolumn{2}{|c|}{$F(3,26)=1,538$} \\
\hline \multicolumn{11}{|l|}{ Modelo 2} \\
\hline Intercepto & 2,4176 & $5,55^{* *}$ & 3,0398 & $3,00^{* *}$ & 0,6495 & 0,92 & 2,5937 & $3,69^{* *}$ & 3,3869 & $3,17^{* *}$ \\
\hline Tamaño grupo & $-0,0145$ & $-0,78$ & $-0,0537$ & 1,24 & $-0,0294$ & $-0,97$ & 0,0577 & $1,92^{*}$ & $-0,0328$ & $-0,72$ \\
\hline GDQ - Fase IV & 0,1217 & 0,89 & 0,3092 & 0,98 & $-0,0982$ & $-0,44$ & $-0,0813$ & $-0,37$ & 0,3572 & 1,07 \\
\hline GDQ & $-0,1626$ & $-0,75$ & $-0,6919$ & $-1,37$ & 0,2820 & 0,80 & 0,1955 & 0,56 & $-0,4366$ & $-0,82$ \\
\hline \multirow[t]{3}{*}{ EDG } & 0,2532 & $2,41^{*}$ & 0,4404 & $1,81^{*}$ & 0,5579 & $3,82^{* *}$ & $-0,1014$ & $-0,60$ & 0,1160 & 0,45 \\
\hline & \multicolumn{2}{|c|}{$\mathrm{R}^{2}$ adj. $=0,402$} & \multicolumn{2}{|c|}{$\mathrm{R}^{2}$ adj. $=0,237$} & $\mathrm{R}^{2}$ adj. $=$ &, 537 & $\mathrm{R}^{2}$ adj. = &, 0633 & $\mathrm{R}^{2}$ adj. = &, 0228 \\
\hline & $F(4,25)=$ & $5,888^{* *}$ & $F(4,25)=$ & $3,252^{* *}$ & $F(4,25)$ & $9,422^{* *}$ & $F(4,25)=$ & 1,49 & $F(4,25)$ & 1,17 \\
\hline
\end{tabular}

$\mathrm{N}=30$ grupos.

${ }^{*} p<0,05,{ }^{* *} p<0,01$. 


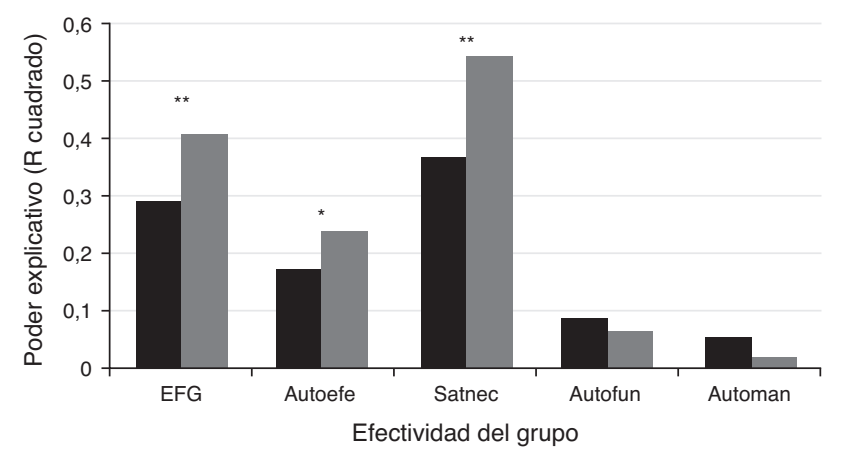

Modelo 1

Modelo 2

Figura 2 Capacidad de predicción de los modelos de regresión. EFG: desempeño del grupo; Autoefe: valoración de eficacia; Satnec: satisfacción de necesidades; Autofun: funcionamiento interno; Automan: mantenimiento del grupo. Modelo 1: Predictores: GDQ-Fase IV y GDQ; Modelo 2: Predictores: GDQFase IV, GDQ y EDG. ${ }^{*} p<0,05,{ }^{* *} p<0,01$.

\section{Discusión}

La presente investigación tuvo como objetivo principal estudiar la relación entre el desarrollo grupal y el desempeño en equipos de proyecto. Los resultados han apuntado, como se esperaba, a que el desarrollo grupal es un determinante importante del desempeño alcanzado por este tipo de equipos. Simplificando, el $40 \%$ del desempeño de estos equipos puede ser explicado por su desarrollo grupal. Este dato es muy similar al encontrado por Navarro et al. (2015) en el que trabajaron con 72 grupos de producción.

\section{Equipos efectivos de proyecto}

Considerando las características particulares de los equipos de proyecto en cuanto a su composición, características de la tarea y mecanismos de integración (Chiocchio y Essiembre, 2009; Sundstrom, McIntire, Halfhill y Richards, 2000; Zwikael y Unger-Aviram, 2010) las relaciones encontradas aquí entre el desarrollo y el desempeño en estos equipos son esperables. Justamente los equipos con un alto desarrollo se caracterizan por una serie de procesos psicosociales (alta interacción entre los miembros, identificación con el colectivo, compartición de recursos, orientación del comportamiento hacia el logro colectivo, etc.) que es razonable presuponer constituyen unos buenos ingredientes para afrontar los proyectos con éxito.

Por otro lado, los equipos de proyecto suelen también caracterizarse por tareas de equipos con una elevada interdependencia (i. e. las tareas requieren de la interacción e intercambio constante entre los miembros) así como elevada incertidumbre (e.g., se desconoce el resultado final, puede haber cambios importantes a lo largo del desarrollo del proyecto, etc.). Estas características de las tareas, interdependencia e incertidumbre, han sido propuestas justamente como un tipo de demandas en las que los equipos altamente desarrollados se manejan con éxito (e.g., Navarro et al., 2011; Wageman, 1995). El desarrollo se constituiría pues en un mediador clave entre las tareas que han de hacer estos equipos y los resultados que finalmente logran.
Según los resultados aquí presentados el desarrollo del grupo tendría una relación especialmente significativa en el desempeño general del equipo, así como en dos de las dimensiones básicas de este, la valoración de eficacia y la satisfacción de necesidades de los miembros. Por el contrario, el desarrollo no ha sido útil para explicar el funcionamiento interno del equipo y el mantenimiento del grupo, los otros dos indicadores de desempeño estudiados. Respecto al funcionamiento interno, considerando otros resultados como el valor bajo de fiabilidad y el patrón de correlaciones, pareciera que la escala no ha funcionado en esta aplicación. Distinta es la situación respecto a la medida de mantenimiento en la que sí contamos con confiabilidad en la medida y correlaciones significativas y esperables con el resto de medidas de desempeño eficaz. Pareciera que en el tipo de equipos con los que hemos trabajado, tal vez por la duración de los mismos (4 meses), el mantenimiento del grupo no se ha visto afectado por el desarrollo. No disponemos de información de un elemento clave en este caso como es la estabilidad de dichos equipos, es decir, con qué frecuencia cambiaban de miembros.

Tampoco ha resultado significativo, en la mayoría de los análisis, el tamaño del grupo. Creemos en este caso que el resultado es explicable por los tamaños considerados en la muestra en las que se trata, en todos los casos, de equipos pequeños, cuando la literatura previa ha estudiado esta variable considerando equipos mayores de, por ejemplo, 10 o más miembros (e.g., Wheelan, 2009) en los que sí se ha comprobado la influencia en la eficacia de este factor. En cualquier caso, y concluyendo, tomando los resultados más significativos podemos afirmar que equipos más desarrollados se valoran a su vez como más efectivos.

\section{Validez Incremental de la escala de desarrollo del grupo respecto al Group Development Questionnaire}

Aunque estamos hablando de desarrollo del grupo hay que precisar en este punto que estamos manejando dos perspectivas teóricas con diferencias importantes. Creemos importante destacar dos de ellas. La primera diferencia es la forma de aproximarse al fenómeno. Mientras que el DG ofrece un enfoque cuantitativo útil para evaluar el grado en que un grupo es un grupo, en función de su grado de desarrollo en las cuatro propiedades básicas (interrelación, identificación, coordinación y orientación a metas colectivas), el GDQ se basa en un enfoque que combina lo cuantitativo con lo cualitativo al concebir la idea de desarrollo como fases cualitativamente distintas por las que pasa un grupo durante su existencia y, a su vez, presuponer que un grupo está más desarrollado en unas fases que en otras. La segunda diferencia es más importante y radica en la propia concepción de desarrollo en ambas propuestas. Para el DG cualquier grupo, con independencia de su etapa de desarrollo, muestra en cierto grado las cuatro propiedades básicas mencionadas. El desarrollo aquí es considerado como un fenómeno continuo respecto al cual cualquier grupo puede ser evaluado. Por su parte, los modelos de fases han propuesto un concepto de desarrollo que combina la continuidad (sucesión entre las fases) con saltos cualitativos en ese desarrollo (los cambios de fase). De esta manera, y a nuestro 
juicio, el DG complementaría desde un enfoque cuantitativo a los enfoques clásicos de fases.

Ahora bien, los resultados en esta investigación con equipos de proyecto han mostrado que DG (a través de la escala de desarrollo grupal) es un mejor predictor del desempeño que los modelos de fases (a través de la medida del GDQ; ver figura 2). De otra forma, que la escala de desarrollo grupal presentaría validez incremental sobre el GDQ y tomando como variable criterio el desempeño de los equipos. La validez incremental se refiere al grado en que una medida explica un fenómeno de interés en relación con otras medidas y, cuándo se trata nuevos instrumentos de evaluación, éstos deben demostrar su capacidad para incrementar la explicación sobre la variable resultado más allá de lo que sean capaces las herramientas ya existentes (LeBreton, Hargis, Griepentrog, Oswald y Ployhart, 2007; Sackett y Lievens, 2008). Esta situación sería, en gran medida, la que nos encontramos aquí, ya que la EDG, sin ser una herramienta nueva, sí que cuenta con un menor uso en comparación con el GDQ debido a su reciente aparición. No obstante, hemos de recordar también que no hemos aplicado el GDQ al completo, al resultar ello imposible por tratarse de un instrumento que nunca se ha comunicado al completo mediante publicaciones. Lo que hemos utilizado ha sido la versión que continuamente ha sido publicada por sus autores (e.g., Buzaglo y Wheelan, 1999; Wheelan, 2009; Wheelan y Hochberger, 1996).

Siguiendo con la comparativa entre EDG y GDQ también es de interés señalar otras dos ventajas, a nuestro juicio, del primero respecto al segundo de los instrumentos. Primera, el EDG permite una evaluación más rápida del desarrollo grupal por contar con un menor número de ítems (en especial si consideramos la versión completa del GDQ con 60 ítems). Y segunda y más importante, uno de los problemas que puede tener el GDQ en su descripción de la cuarta de las fases es la de cierta tautología con medidas justamente de desempeño. Si prestamos atención a los ítems que conforman la escala de la Fase IV (e.g., «El grupo recibe, da y utiliza retroalimentación sobre su efectividad y productividad», «El grupo ejecuta sus decisiones», «Este grupo estimula el alto rendimiento y el trabajo de alta calidad») podemos acordar que son en gran parte ítems que también podrían considerarse como una medida de desempeño. Esto no ocurriría en el caso de la medida EDG en la se recoge información sobre características básicas de todo grupo (e.g., interacción, identificación, orientación al colectivo, etc.) que creemos claramente no corresponden con una medida de desempeño. Se evitaría, por tanto, la tautología mencionada.

\section{Contribuciones prácticas y académicas}

Según nuestro estudio, y otros previos que hemos recogido, una de las vías para aumentar la efectividad de los equipos de proyectos es incrementando el desarrollo grupal. Para conseguir equipos efectivos de proyecto hay que conseguir, previamente, que los miembros interaccionen regularmente (diseñando encuentros frecuentes de todo el grupo, por ejemplo), se identifiquen con el grupo (trabajando en la generación de una identidad social específica del grupo), se coordinen (diseñando tareas interdependientes, por ejemplo) y orienten sus esfuerzos hacia la consecución de metas colectivas (recompensando estos comportamientos de inte- rés para todo el grupo, por ejemplo). El diseño y desarrollo de equipos, como herramienta ampliamente conocida (e.g., Salas, 2015; West, 2012), se presenta clave para la generación de equipos de éxito.

Por otro lado, otro de los aportes del presente estudio está asociado con las características de la herramienta EDG. La escala de desarrollo del grupo es de fácil aplicación, no requiere entrenamiento especial excepto el relativo al manejo en procesos típicos de evaluación organizacional a través de encuestas, requiere poco tiempo para su aplicación y es de bajo coste. En la aplicación del GDQ original es preciso tener en cuenta la formación adicional que conlleva, con los costes asociados tanto económicos como de tiempo; en la versión reducida aquí utilizada, el instrumento está en igualdad de condiciones que la EDG, pero esta última, según los resultados obtenidos, ofrece mayor información predictiva del desempeño eficaz grupal. Se trata, por tanto, de un aporte significativo pues nos permite ofrecer una herramienta desarrollada en español, fundamentada en planteamientos provenientes de líneas teóricas de reconocida trayectoria, avalada por datos empíricos obtenidos de equipos reales y aplicada en diferentes contextos organizacionales.

\section{Limitaciones y perspectivas futuras}

Aunque la presente investigación ha contribuido a la comprensión de la relación entre el desarrollo grupal en los equipos de trabajo y su desempeño eficaz, para efectos de generalización de los resultados se precisa cautela. Haber utilizado un diseño transversal, así como el uso de una muestra pequeña, representan limitaciones sobre esa validez externa. Por otro lado, y como es común en parte de la investigación generada en el área, el uso de un mismo tipo de instrumentos de medida atenta contra la validez interna al incrementarse la varianza debida al uso de un método común. En cuanto a la comparación realizada entre las dos herramientas de evaluación del desarrollo grupal, la EDG y el GDQ, es pertinente acotar nuevamente que se utilizó una versión reducida del GDQ original, por inaccesibilidad a la herramienta completa para efectos de investigación.

Para futuras investigaciones sería deseable llevar a cabo estudios longitudinales, con muestras más amplias compuestas por diferentes tipos de equipos de trabajo, ya que según la literatura los diferentes tipos de equipos no necesariamente se comportan igual. Igualmente, si se trabaja con equipos de proyectos sería recomendable tener en cuenta la duración del proyecto como variable moderadora de la relación entre el desarrollo del equipo y el éxito del proyecto (Zwikael y Unger-Aviram, 2010). Por último, la consideración del tipo de tarea, así como el incluir criterios de efectividad (i. e., de resultados finales conseguidos por el equipo) más allá de las medidas de desempeño aquí manejadas, contribuiría también de manera significativa a generar un mayor conocimiento sobre la relación entre procesos y desempeño en los equipos.

\section{Conclusiones}

Avanzar en la línea de investigación sobre desarrollo grupal y el desempeño de los equipos en las organizaciones propor- 
ciona a gerentes de proyecto, consultores e investigadores información valiosa para comprender los equipos y grupos de trabajo, generar técnicas y diseñar intervenciones orientadas a incrementar la madurez de estos. Con ello se incide en su desempeño. Es imposible obviar que el desempeño eficaz de los grupos y equipos de trabajo es una variable afectada por diversos elementos. Aquí hemos estudiado cómo los procesos, el desarrollo grupal en concreto, es un elemento clave para lograr equipos efectivos de proyecto. $Y$ para evaluar este proceso crítico contamos con herramientas, como la EDG, breve y útil, que también nos ayudará, por tanto, para orientar posibles intervenciones.

\section{Bibliografía}

Arrow, H., McGrath, J. E. y Berdhal, J. L. (2000). Small groups as complex systems. Formation, coordination, development and adaptation. Thousand Oaks, CA: Sage.

Buzaglo, G. y Wheelan, S. (1999). Facilitating work team effectiveness. Case studies from Central America. Small Group Research, 30, 108-129

Cohen, A., Doveh, E. y Nahum-Shani, I. (2009). Testing agreement for multi-item scales with the indices $\operatorname{rwg}(\mathrm{j})$ and $\operatorname{adm}(\mathrm{j})$. Organizational Research Methods, 12, 148-164.

Chang, A., Bordia, P. y Duck, J. (2003). Punctuated equilibrium and linear progression: Toward a new understanding of group development. Academy of Management Journal, 46, 106-117.

Chang, A., Duck, J. y Bordia, P. (2006). Understanding the multidimensionality of Group Development. Small Group Research, 37, 327-350.

Chiocchio, F. y Essiembre, H. (2009). Cohesion and performance: A meta-analytic review of disparities between project teams, production teams, and service teams. Small Group Research, 40, 382-420.

Gersick, C. J. G. (1988). Time and transition in work teams: Toward a new model of group development. Academy of Management Journal, 31, 9-41.

Gersick, C. J. G. (1989). Marking time: Predictable transitions in tasks groups. Academy of Management Journal, 32, 274-309.

Gully, S. M., Incalcaterra, K. A., Joshi, A. y Beaubien, J. M. (2002). A meta-analysis of team-efficacy, potency, and performance: Interdependence and level of analysis as moderators of observed relationships. Journal of Applied Psychology, 87, 819-832.

Hackman, J. R. (1987). The design of work teams. En J. W. Lorsch (Ed.), Handbook of organizational behavior (pp. 315-342). Englewood Cliffs, NJ: Prentice-Hall.

Hunsley, J. y Meyer, G. J. (2003). The incremental validity of psychological testing and assessment: Conceptual, methodologi$\mathrm{cal}$, and statistical issues. Psychological Assessment, 15, 446-455.

LeBreton, J. M., Hargis, M. B., Griepentrog, B., Oswald, F. L. y Ployhart, R. (2007). A multidimensional aproach for evaluating variables in organizational research and practice. Personnel Psychology, 60, 475-498.

LePine, J. A., Piccolo, R. F., Jackson, C. L., Mathieu, J. E. y Saul, J.R. (2008). A meta-analisys of teamwork processes: test of a multidimensional model and relationships with team effectiveness criteria. Personnel Psychology, 61, 273-307.

Lickel, D., Hamilton, D., Lewis, A., Sherman, S., Wieczorkowska, G. y Uhles, A. N. (2000). Varieties of groups and the perception of group entitativity. Journal of Personality and Social Psychology, 78, 223-246.
Marks, M. A., Mathieu, J. E. y Zaccaro, S. J. (2001). A temporally based framework and taxonomy of team processes. Academy of Management Review, 26, 356-376.

McGrath, J. E. (1984). Groups: Interaction and performance. Inglewood, N. J.: Prentice Hall, Inc.

McGrath, J. E. y Argote, L. (2001). Group processes in organizational context. En M. A. Hogg y R. S. Tindale (Eds.), Blackwell handbook of social psychology: Group processes (pp. 603-627). Oxford: Blackwell.

Meneses, R. y Navarro, J. (2015). ¿Cómo mejorar la eficacia de los equipos a través de los procesos grupales? Un ejemplo en la industria automotriz. Papeles del Psicólogo, 36, 224-229.

Meneses, R., Ortega, R., Navarro, J. y De Quijano, S. D. (2008). Criteria for assessing the level of group development (LGD) of work groups. Groupness, entitativity, and groupality as theoretical perspectives. Small Group Research, 39, 492-514.

Navarro, J., Meneses, R., Miralles, C., Moreno, D. y Loureiro, V. (2015). What distinguish teams from social aggregates? A tool to assess the level of group development. Anales de Psicología, 31, 921-929.

Navarro, J., Quijano, S. D., Berger, R. y Meneses, R. (2011). Grupos en las organizaciones: Herramienta básica para gestionar la incertidumbre y ambigüedad crecientes. Papeles del Psicólogo, 32, 17-28.

Rico, R., Alcover, C. M. y Tabernero, C. (2010). Efectividad de los equipos de trabajo, una revisión de la última década de investigación (1999-2009). Revista de Psicología del Trabajo y de las Organizaciones, 26, 47-71.

Sackett, P. R. y Lievens, F. (2008). Personnel selection. Annual Review of Psychology, 59, 419-450.

Salas, E. (2015). Team training essentials. A research-based gruide. London, UK: Taylor \& Francis Ltd.

Srivastava, A., Bartol, K. M. y Locke, E. A. (2006). Empowering leadership in management teams: Effects on knowledge sharing, efficacy, and performance. Academy of Management Journal, 49, 1239-1251.

Stewart, G. L. (2006). A meta-analytic review of relationships between team design features and team performance. Journal of Management, 32, 29-55.

Sundstrom, E., McIntire, M., Halfhill, T. y Richards, H. (2000). Work groups: from Hawthorne studies to work teams of the 1990s and beyond. Group Dynamics: Theory, Research and Practice, 4, 44-67.

Tuckman, B. W. y Jensen, M. A. (1977). Stages of small-group development revisited. Group \& Organization Studies, 2, 419-427.

Wageman, R. (1995). Interdependence and group effectiveness. Administrative Science Quarterly, 40, 145-180.

West, M. (2012). Effective teamwork. Practical lessons from organizational research. London: UK: Blackwell.

Wheelan, S. (1994). Group processes: A development perspective. Boston: Allyn \& Bacon.

Wheelan, S. (1999). Introduction to this special issue on Group Development. Small Group Research, 30, 3-7.

Wheelan, S. (2005). Group processes: A developmental perspective (2nd ed.). Boston: Allyn \& Bacon.

Wheelan, S. (2009). Group size, group development and group productivity. Small Group Research, 40, 247-262.

Wheelan, S., Davidson, B. y Tilin, F. (2004). Group development across time: Reality or illusion? Small Group Research, 34, 223245.

Wheelan, S. y Hochberger, J. (1996). Validation studies of the group development questionnaire. Small Group Research, 27, 143-170

Zwikael, O. y Unger-Aviram, E. (2010). HRM in project groups: The effect of project duration on team development effectiveness. International Journal of Project Management, 28, 413-421. 